\title{
IncRNA GAS5, as a ceRNA, inhibits the proliferation of diffuse large B-cell lymphoma cells by regulating the miR-18a-5p/RUNX1 axis
}

\author{
YINSHA MIAO ${ }^{1-3}$, XIAODONG CHEN ${ }^{1}$, MENGTING QIN ${ }^{1}$, WEN ZHOU ${ }^{1}$, YANG WANG ${ }^{1}$ and YANHONG JI $^{1}$ \\ ${ }^{1}$ Department of Pathogenic Biology and Immunology, School of Basic Medical Sciences, \\ Xi'an Jiaotong University Health Science Center, Xi'an, Shaanxi 710061; ${ }^{2}$ Department of Clinical Laboratory, \\ ${ }^{3}$ Xi'an Key Laboratory of Cardiovascular and Cerebrovascular Diseases, The Affiliated Hospital of Northwest University, \\ Xi'an No. 3 Hospital, Xi'an, Shaanxi 710018, P.R. China
}

Received May 30, 2021; Accepted September 29, 2021

DOI: $10.3892 /$ ijo.2021.5274

\begin{abstract}
Diffuse large B-cell lymphoma (DLBCL) is a common and fatal malignant tumor caused by B-lymphocytes. Long non-coding RNA (lncRNA) GAS5 (growth arrest specific 5) has been reported to function as a tumor suppressor gene, and is differentially expressed in DLBCL. The present study aimed to explore the potential mechanisms of action of lncRNA GAS5 in the proliferation of DLBCL cells. The expression levels of GAS5, miR-18a-5p and Runt-related transcription factor 1 (RUNX1) in DLBCL cell lines were detected using reverse transcription-quantitative polymerase chain reaction, and their effects on cell proliferation, the cell cycle and apoptosis were determined using 5-ethynyl-2'-deoxyuridine assay and flow cytometry. Dual-luciferase reporter and RNA pull-down assays were used to evaluate the interaction between GAS5 and miR-18a-5p, or between miR-18a-5p and RUNX1. Chromatin immunoprecipitation assay was used to identify the interaction between RUNX1 and BAX. The expression levels of GAS5 and RUNX1 were downregulated; however, miR-18a-5p expression was upregulated in the DLBCL cell lines compared with the normal controls. GAS5 directly interacted with miR-18a-5p by acting as a competing endogenous RNA (ceRNA) and reversed the low expression of RUNX1 induced by miR-18a-5p. Additionally, the knockdown of RUNX1 reversed the inhibitory effects of GAS5 on the proliferation and cell cycle G1 arrest, and its promoting effects on the apoptosis of OCI-Ly3 and TMD8 cells. Moreover,
\end{abstract}

Correspondence to: Dr Yanhong Ji, Department of Pathogenic Biology and Immunology, School of Basic Medical Sciences, Xi'an Jiaotong University Health Science Center, 76 Yanta West Road, Xi'an, Shaanxi 710061, P.R. China

E-mail: jiyanhong@xjtu.edu.cn

Key words: lncRNA growth arrest specific 5, diffuse large B-cell lymphoma, proliferation, miR-18a-5p, Runt-related transcription factor 1
RUNX1 enhanced BAX expression by directly binding to the BAX promoter. On the whole, the present study demonstrates that GAS5 functions as a ceRNA, inhibiting DLBCL cell proliferation by sponging miR-18a-5p to upregulate RUNX1 expression. These findings may provide a potential therapeutic strategy for DLBCL.

\section{Introduction}

Diffuse large B-cell lymphoma (DLBCL) is a type of lymphoid malignancy that accounts for $25-35 \%$ of non-Hodgkin's lymphoma (NHL) and $37 \%$ of B-cell tumors (1). Germinal center B-cell-like (GCB) and activated B-cell-like (ABC) are the two main molecular subtypes of DLBCL (2). DLBCL occurs in lymph node or extranodal structures and is pathologically characterized by a diffuse growth pattern of large B-cell proliferation that replaces these sites (3). Due to its lack of obvious symptoms in the early stages and similar symptoms to other diseases, it is often characterized by evident heterogeneity and complexity, which is also one of the main reasons for the poor prognosis and the low 5-year survival rate (30-80\%) of patients $(4,5)$. Therefore, the exploration of novel therapeutic targets for DLBCL is of utmost importance.

Long non-coding RNAs (lncRNAs) refer to RNA molecules of $>200$ nucleotides in length that do not encode proteins. There is evidence to indicate that lncRNAs play an important role in malignant B-cells and serve as potential markers for the diagnosis and progression of DLBCL; thus, they may play carcinogenic or tumor suppressive functions in the DLBCL process. For example, IncRNA MALAT1 (6), lncRNA FIRRE (7) and lncRNA NEAT1 (8) have been shown to promote tumorigenesis, and conversely, lncRNA SMAD5-AS1 (9), lncRNA PANDA (10) and lncRNA RP11-468E2.5 (11) have been shown to inhibit tumor function. Previous studies have indicated that lncRNA growth arrest specific 5 (GAS5) is abnormally expressed in colorectal cancer, gastric cancer, glioma and other types of cancer, and functions as a tumor suppressor gene (12-15). Moreover, GAS5 has been found to be associated with cell survival and with the progression of B-cell lymphoma (16). Previous studies have 
also discovered that GAS5 is abnormally expressed in patients with DLBCL, indicating its involvement in the pathogenesis of DLBCL $(17,18)$. Hence, the identification of the molecular function and the biological targets of GAS5 may prove to be helpful for attenuating the progression of DLBCL.

MicroRNAs (miRNAs or miRs) are small single-stranded RNAs that play an important regulatory role by regulating target gene transcription. miR-18a-5p has been demonstrated to play an oncogenic role in lung, nasopharyngeal, prostate, colorectal and breast cancers, and is widely involved in cell proliferation, apoptosis and other phenotypes (19-23). The upregulated expression of miR-18a-5p was previously predicted using a database of differentially expressed miRNAs in human cancers v2.0 (dbDEMC 2.0) in lymphoma and its high expression level in DLBCL tissue was show to potentially play a carcinogenic role (24). Moreover, a previous study confirmed that the expression of miR-18a-5p was also elevated in B-cell lymphoma samples (25). In addition, GAS5 has been shown to target and regulate the expression of miR-18a-5p in cancers cells (26). However, the molecular function of miR-18a-5p in DLBCL remains unclear.

The transcription factor, Runt-related transcription factor 1 (RUNX1), also known as acute myeloid leukemia 1 (AML1), is involved in regulating the development of hematopoietic stem cells and is closely related to the occurrence and development of hematological malignancies (27). Furthermore, a previous study demonstrated that RUNX1 specifically regulated the transcriptional activity of its target gene Ebf1, and participated in B-lymphocyte development (28). Of note, a previous study by the authors demonstrated that miR-18a-5p targeted the regulation of RUNX1 and then participated in the permeability of the blood-tumor barrier (29). However, whether the interaction among GAS5, miR-18a-5p and RUNX1 affects the biological behavior of DLBCL cells and plays a carcinogenic role remains to be further investigated.

The present study aimed to determine the expression levels of GAS5, miR-18a-5p and RUNX1 in DLBCL cell lines, and to further investigate the potential molecular mechanisms among them in DLBCL. The findings presented herein may provide novel therapeutic targets for DLBCL.

\section{Materials and methods}

Cell line culture and transfection. The ABC DLBCL cell line (OCI-Ly3, BNCC338435), GCB DLBCL cell line (TMD8, BNCC340121) (9) and the human B-lymphocyte (GM12878, BNCC341257) were all purchased from the American Type Culture Collection (ATCC). The Burkitt's lymphoma cell line, Raji, was purchased from The Cell Bank of Type Culture Collection of the Chinese Academy of Sciences (TCHu 44). The cells were cultured in the Iscove's modified Dulbecco's medium (IMDM) supplemented with $20 \%$ fetal bovine serum, $100 \mathrm{U} / \mathrm{ml}$ penicillin and $100 \mu \mathrm{g} / \mathrm{ml}$ streptomycin in $5 \% \mathrm{CO}_{2}$ at $37^{\circ} \mathrm{C}$. The cells were digested by trypsin and spread on a $24-w e l l$ plate, and further cultured in IMDM (Gibco; Thermo Fisher Scientific, Inc.) in an incubator at $37^{\circ} \mathrm{C}$ for $2-3$ days. The $2 \mu \mathrm{g}$ lentiviral vector containing the GAS5 sequence (Lv-GAS5) was purchased from Shanghai GenePharma Co., Ltd. and transfected into the OCI-Ly3/TMD8 cells with $10 \mu \mathrm{g} / \mu \mathrm{l}$ polybrene [40804ES76, 1:1,000; Yeasen Biotechnology (Shanghai) Co.,
Ltd.] to construct GAS5-overexpressing cells. In addition, cells transfected with the $20 \mathrm{nmol} / \mathrm{l}$ empty vector (GenePharma) were considered as Lv. Other transfection targets, including a total of $25 \mathrm{pmol} / 1 \mathrm{miR}-18 \mathrm{a}-5 \mathrm{p}$ mimics/inhibitor, $100 \mathrm{nmol} / \mathrm{l}$ si-RUNX1/RUNX1 overexpression plasmid (pcDNA3.1) (Sangon Biotech Co., Ltd.) and their negative controls (NC) were transfected into the cells using Lipofectamine $3000^{\circledR}$ transfection reagent (Invitrogen; Thermo Fisher Scientific, Inc.) at room temperature for $48 \mathrm{~h}$, respectively. The cells were collected for subsequent experiments at $48 \mathrm{~h}$ following transfection. Cells without transfection were considered as controls. The sequences of the transfection targets are presented in Table I.

Reverse transcription-quantitative polymerase chain reaction $(R T-q P C R)$. TRIzol ${ }^{\circledR}$ reagent $(500 \mu 1)$ (Sangon Biotech Co., Ltd.) was used to extract the total RNA, and 500 ng RNA were used to synthesize cDNA for reverse transcription using the 5X Prime Script RT Master Mix (Takara Bio, Inc.). The cDNA (1 $\mu \mathrm{l}$ ) was used as the template for RT-qPCR using SYBR qPCR Master Mix (Takara Bio, Inc.) according to the manufacturer's protocol. The PCR reaction conditions were $95^{\circ} \mathrm{C}$ for $5 \mathrm{~min}, 95^{\circ} \mathrm{C}$ for $30 \mathrm{cycles}, 60^{\circ} \mathrm{C}$ for $30 \mathrm{sec}, 72^{\circ} \mathrm{C}$ for $30 \mathrm{sec}$, and finally $72^{\circ} \mathrm{C}$ for $7 \mathrm{~min}$. The expression levels were normalized to the glyceraldehyde-3-phosphate dehydrogenase (GAPDH) levels and analyzed using the $2^{-\Delta \Delta \mathrm{Cq}}(30)$ method. The primer sequences used are presented in Table II.

RNA fluorescence in situ hybridization (FISH). The location of GAS5 in two cell lines (OCI-Ly3 and TMD8) was identified using the Ribo ${ }^{\mathrm{TM}}$ FISH kit (Guangzhou RiboBio Co., Ltd.) according to the manufacturer's instructions. Briefly, $6 \times 10^{4}$ cells/well were fixed with $4 \%$ paraformaldehyde for $10 \mathrm{~min}$ at room temperature, followed by permeabilization with $0.5 \%$ Triton, and washing three times with phosphate-buffered saline (PBS) after discarding the permeabilization solution. Each well was then supplemented with a pre-hybridization solution (Reagent A, derived from the RiboBio FISH kit) and blocked with blocking solution (Reagent $\mathrm{C}$, derived from the RiboBio FISH kit) at $37^{\circ} \mathrm{C}$ for $30 \mathrm{~min}$. Cy3-labeled lncRNA FISH probes (included with the kit) were then synthesized and used to identify GAS5. The probe mix $(2.5 \mu \mathrm{l}$ and $20 \mu \mathrm{M})$ was added to $100 \mu$ l hybridization solution (Reagent $\mathrm{B}$, derived from the RiboBio FISH kit), and then mixed and added to the cells, followed by hybridization overnight at $37^{\circ} \mathrm{C}$. Furthermore, the cells were washed three times with hybridization washing solution, and then the nuclei were stained with DAPI (Reagent D, derived from the RiboBio FISH kit) for $10 \mathrm{~min}$ at room temperature, and fixed on a glass slide for fluorescence detection. The aforementioned process was carried out in a dark environment. Focus was placed on five fields of view, and the cells were observed under a TCS SP5II confocal microscope (Leica Microsystems GmbH).

Cell viability and proliferation assay. Cell viability was assessed using the 3-(4,5-dimethylthiazol-2-yl)-5-(3-carbox ymethoxyphenyl)-2-(4-sulfophenyl)-2H-tetrazolium (MTS) kit (ab197010; Abcam). Briefly, the cells were seeded in a 96-well plate at $5.0 \times 10^{3} /$ well, and $20 \mu \mathrm{l}$ MTS solution were then added and incubated in an incubator at $37^{\circ} \mathrm{C}$ for $3 \mathrm{~h}$. 
Table I. Sequences of the transfection targets in the present study.

\begin{tabular}{|c|c|}
\hline Gene & Sequences (5' to $\left.3^{\prime}\right)$ \\
\hline $\begin{array}{l}\operatorname{miR}-18 a-5 p \\
\text { mimics }\end{array}$ & UAAGGUGCAUCUAGUGCAGAUAG \\
\hline NC mimics & UUCUCCGAACGUGUCACGUTT \\
\hline $\begin{array}{l}\text { miR-18a-5p } \\
\text { inhibitor }\end{array}$ & CCCUAUCUGCACUAGAUGCACCU \\
\hline NC inhibitor & CAGUACUUUUGUGUAGUACAA \\
\hline si-RUNX1 & ACGAATCACACTGAATGCAAACC \\
\hline si-NC & TGCTTAGTGTGACTTACGTTTGG \\
\hline
\end{tabular}

NC, negative control; RUNX1, Runt-related transcription factor 1 .

The absorbance at $450 \mathrm{~nm}$ was detected using a microplate analyzer (Tecan Group, Ltd.). Cell proliferation was measured using a 5-ethynyl-2'-deoxyuridine (EdU) kit (C0085S; Beyotime Institute of Biotechnology) following the manufacturer's instructions. Firstly, 2X EdU working solution $(20 \mu \mathrm{M})$ were added to the 6-well plate and incubated for $4 \mathrm{~h}$ at $37^{\circ} \mathrm{C}$. Following centrifugation at $1,000 \mathrm{x}$ for $5 \mathrm{~min}$ at room temperature, $1 \mathrm{ml}$ of PBS was added to resuspend the cells and cell suspension drops were added to the slide and spread evenly, and further placed in an oven at $50^{\circ} \mathrm{C}$ for 1 $\mathrm{h}$ and fixed with 4\% paraformaldehyde (P0099; Beyotime Institute of Biotechnology) at room temperature for $1 \mathrm{~min}$. Subsequently, the cells were treated with washing solution (P0106; Beyotime Institute of Biotechnology) and permeability solution (P0097, Beyotime Institute of Biotechnology); the click reaction solution (derived from the EdU kit) was then added to evenly cover the cell surface followed by incubated at room temperature for $30 \mathrm{~min}$ in the dark environment. Finally, $1 \mathrm{ml} 1 \mathrm{X}$ Hoechst 33342 solution (C1025, Beyotime Institute of Biotechnology) was used to incubate the cells for $30 \mathrm{~min}$ at room temperature and avoid lighting for $10 \mathrm{~min}$. After washing, the fluorescence detection was performed.

Cell cycle assay. The cells were collected by centrifugation at $1,000 \mathrm{x} \mathrm{g}$ for $5 \mathrm{~min}$ at $4^{\circ} \mathrm{C}$. Subsequently, cells were fixed with $1 \mathrm{ml}$ pre-cooled $70 \%$ ethanol overnight at $4^{\circ} \mathrm{C}$. Propidium iodide (PI) staining solution $(0.5 \mathrm{ml})$ was then added to each tube of cell samples for resuspension, followed by incubation at $37^{\circ} \mathrm{C}$ in a dark environment for $30 \mathrm{~min}$. Finally, the red fluorescence and light scattering at the excitation wavelength of $488 \mathrm{~nm}$ were detected using a flow cytometer (FACScan; BD Biosciences). The data were analyzed using FlowJo 10 software.

Cell apoptosis assay. The number of apoptotic cells was detected using flow cytometry. Firstly, the cells were washed twice with pre-cooled PBS and centrifuged at $300 \mathrm{x}$ g for $5 \mathrm{~min}$ at $4^{\circ} \mathrm{C}$. Following PBS absorption, $100 \mu \mathrm{l} 1 \mathrm{X}$ binding buffer (Nanjing KeyGen Biotech Co., Ltd.) were added to resuspend the cells. Subsequently, $5 \mu 1$ Annexin V-FITC and $10 \mu 1$ PI staining solution (Beyotime Institute of Biotechnology) were added and mixed gently, while being protected from light and reacted for 10-15 $\mathrm{min}$ at room temperature. Finally, $400 \mu \mathrm{l}$
Table II. Primer sequences used in RT-qPCR in the present study.

\begin{tabular}{ll} 
Primers & \multicolumn{1}{c}{ Sequences (5' to 3') } \\
\hline $\begin{array}{l}\text { lncRNA-GAS5-Forward } \\
\text { lncRNA-GAS5-Reverse }\end{array}$ & GCAAGCCTAACTCAAGCCATTG \\
RUNX1-Forward & CCTCAGGTTTGTCGGTCGAA \\
RUNX1-Reverse & CTTGCGGTGGGTTTGTGAAG \\
BAX-Forward & CATGGGCTGGACATTGGACT \\
BAX-Reverse & CAAAGTAGGAGAGGAGGCCG \\
miR-18a-5p-RT & GTCGTATCCAGTGCAGGGTCCGAG \\
& GTATTCGCACTGGATACGACctatct \\
miR-18a-5p-Forward & CGTTATAAGGTGCATCTAGTGC \\
miR-18a-5p-Reverse & GTGCAGGGTCCGAGGT \\
U6-RT & AACGCTTCACGAATTTGCGT \\
U6-Forward & CTCGCTTCGGCAGCACA \\
U6-Reverse & AACGCTTCACGAATTTGCGT \\
GAPDH-Forward & GTTCGTCATGGGTGTGAACC \\
GAPDH-Reverse & CATCCACAGTCTTCTGGGTG
\end{tabular}

GAS5, growth arrest specific 5; RUNX1, Runt-related transcription factor 1; $\mathrm{RT}$, reverse transcription.

$1 \mathrm{X}$ binding buffer were added, mixed and placed on ice, and subsequently detected using flow cytometry (FACScan; BD Biosciences) within $1 \mathrm{~h}$. The data were analyzed using FlowJo 10 software.

Luciferase reporter assay. The wild-type and mutant-type 3'UTR sequences of RUNX1 were inserted into the pmirGLO dual luciferase reporter vector (E1330; Promega Corporation) to construct wild-type (WT) and mutated-type (MUT) luciferase reporter plasmids. The luciferase plasmid (200 ng) was then combined with $60 \mathrm{nM}$ miR-18a-5p mimics/inhibitor and co-transfected into OCI-Ly3 cells. Lipofectamine $2000^{\circledR}$ reagent (Invitrogen; Thermo Fisher Scientific, Inc.) was used for transfection. Following transfection, the cells were washed with pre-cooled PBS, and then lysed using a dual luciferase reporter gene detection kit (E1960; Promega Corporation). Subsequently, $30 \mu \mathrm{l}$ Firefly luciferase detection reagent were added to the lysis solution, and the relative light unit (RLU) was measured using a multifunctional microplate reader (Fluoroskan ascent FL, Thermo Fisher Scientific, Inc.). Renilla luciferase detection solution $(30 \mu \mathrm{l})$ was then added to the lysate for RLU determination. Finally, the relative luciferase activity was calculated (E1960; Promega Corporation).

Western blot analysis. The cells were washed with pre-cooled PBS and the culture plate was then placed on ice. Subsequently, $10 \mu \mathrm{l}$ phenylmethanesulfonyl fluoride (PMSF) were added followed by lysis on ice for $30 \mathrm{~min}$, centrifugation at $15,000 \mathrm{x}$ g for $5-10 \mathrm{~min}$ at $4^{\circ} \mathrm{C}$ and storage at $-20^{\circ} \mathrm{C}$. The protein extracts were separated through $10 \%$ sodium dodecyl sulphate-polyacrylamide gel electrophoresis (SDS-PAGE) and then transferred onto a polyvinylidene fluoride (PVDF) membrane. The membrane was then rinsed with Tris-buffered saline with Tween-20 (TBST) for $5 \mathrm{~min}$ and transferred to a $5 \%$ skimmed milk powder blocking solution, and then sealed 
on a shaker at room temperature for $2 \mathrm{~h}$. Primary antibodies against RUNX1 (DF6785, 1:1,000; Affinity Biosciences), BAX (AF0120, 1:1,000; Affinity Biosciences) and GAPDH (P04406, 1:1,000; Affinity Biosciences) were added to the membrane and incubated overnight at $4^{\circ} \mathrm{C}$. The diluent of the secondary antibody goat anti-rabbit IgG H\&L (HRP) (S0001, 1:5,000; Affinity Biosciences) was then added to the membrane and incubated for $1 \mathrm{~h}$ at room temperature. After the incubation was completed, TBST was used for rinsing three times for $5 \mathrm{~min}$ at room temperature each time. Enhanced chemiluminescence reagent (Amersham Biosciences) and ImageJ software (version 1.48; National Institutes of Health) were used to detect protein bands and to analyze the optical density. GAPDH was used as a loading control.

RNA antisense purification (RAP) and RNA pull-down assay. The binding effect of GAS5 and miR-18a-5p was detected using the RAP kit (Bes5103; BersinBio) and RNA pull down kit (Thermo Fisher Scientific, Inc.). The biotin-labeled GAS5 probe (Guangzhou RiboBio Co., Ltd.) was combined with the connection region of GAS5, and the oligonucleotide probe (Guangzhou RiboBio Co., Ltd.) was used as a control. The probe was added to the lysed cells in proportion, and incubated with a vertical mixer for $5 \mathrm{~h}$ at room temperature. Subsequently, the RNA enriched on the magnetic beads (Thermo Fisher Scientific, Inc.) was washed multiple times with RNA elution buffer, and the miRNA bound in the complex was then extracted using TRIzol reagent (R0016, Beyotime Institute of Biotechnology) and quantitatively analyzed using RT-qPCR. Subsequently, $50 \mathrm{nmol} / 1$ biotin-labeled miR-18a-5p was transfected into the OCI-ly3 cells for $48 \mathrm{~h}$, and the cells were then lysed with $0.1 \%$ NP-40 (P0013F; Beyotime Institute of Biotechnology), centrifugation at $12,000 \mathrm{x}$ f for $5 \mathrm{~min}$ at $4^{\circ} \mathrm{C}$. incubated for $1 \mathrm{~h}$. The combination of $500 \mu \mathrm{g}$ streptavidin magnetic beads (HY-K0208, MedChemExpress) and 200 pmol biotin-labeled miR-18a-5p (Guangzhou RiboBio Co., Ltd.) were added to the RNA and mixed gently at room temperature, incubated for $30 \mathrm{~min}$ at room temperature. Biotin-scramble was used as a negative control for biotin labeled miR-18a-5p, and the experimental operation was consistent with the above. Elution buffer (Thermo Fisher Scientific) was added to collect the RNA complex pulled down, and this was then analyzed using RT-qPCR as described above.

Chromatin immunoprecipitation (ChIP). The protein-gene interactions were identified using ChIP assay with the Simple Chip Enzymatic Chromatin IP kit (9002S; Cell Signaling Technology, Inc.). The cells were treated with formaldehyde and incubated at $37^{\circ} \mathrm{C}$ for $15 \mathrm{~min}$, and cross-linking was terminated using glycine. The cells were washed and centrifuged at 1,000 $\mathrm{x} g$ for $5 \mathrm{~min}$ at room temperature, and $2 \mathrm{ml}$ cell lysis buffer were then added to resuspend cells. Subsequently, the cells were placed on ice for incubation for $15 \mathrm{~min}$ for lysis, and were finally sonicated to share DNA to an average size of $500 \mathrm{bp}$. The chromatin solution was cleaned with protein A-agarose beads (Cell Signaling Technology, Inc) and incubated overnight with normal IgG (ab172730, $5 \mu \mathrm{g}$; Abcam) or anti-RUNX1 antibody (ab272456,5 $\mu \mathrm{g}$; Abcam) at $4^{\circ} \mathrm{C}$. After the incubation was complete, protein $\mathrm{A}$-agarose beads were added and inverted for $2 \mathrm{~h}$ at $4^{\circ} \mathrm{C}$. Protein $\mathrm{G}$-agarose beads were used to capture the chromatin-immune complex, and the immune complex was eluted with an elution buffer containing $1 \%$ SDS and $0.1 \mathrm{M} \mathrm{NaHCO}_{3}$ (Cell Signaling Technology, Inc). $\mathrm{NaCl}$ and proteinase $\mathrm{K}$ were then mixed and used to reverse cross-linking at $65^{\circ} \mathrm{C}$ for $1 \mathrm{~h}$. The interaction of RUNX1 and the binding region was analyzed using $\mathrm{RT}-\mathrm{qPCR}$. The primers sequences were as follows: R1 forward, CCTGTAATCCCAGCACTTTG and reverse, CTCGGCTTACTGCAACCTCTG; and R2 forward, CAAGGTCACGATCTCAGCTC and reverse, CTGTAATCCCAGCACTTTGGG.

Bioinformatics prediction. The targeted miRNA of lncRNA GAS5 was predicted using online tools, including starBase (http://starbase.sysu.edu.cn/), DIANA (http://carolina.imis. athena-innovation.gr/diana_tools/web/index.php) and NPInter (http://bigdata.ibp.ac.cn/npinter4/browse/). The potential mRNA targets of miR-18a-5p was predicted using online tools, including TargetScan (http://www.targetscan.org/vert_72/), PicTar (https://pictar.mdc-berlin.de/), TarBase (http://www. microrna.gr/tarbase) and microT_CDS (http://www.microrna. gr/microT-CDS). The transcription factor binding sites were predicted by JASPAR (http://jaspar.genereg.net/) software.

Statistical analysis. GraphPad Prism 8.0 (GraphPad Software, Inc.) was used for statistical analysis. These data are presented as the mean \pm standard deviation (SD). One-way ANOVA (followed by Tukey's test) and an unpaired t-test were used for the determination of significant differences between groups. The experiments were carried out independently three times under the same conditions. $\mathrm{P}<0.05$ was considered to indicate a statistically significant difference.

\section{Results}

GAS5 expression is downregulated in DLBCL cell lines. The expression of GAS5 in three DLBCL cell lines (Raji, OCI-ly3 and TMD8) and a human normal lymphocyte cell line (GM12878) was detected using RT-qPCR. The results revealed that the expression level of GAS5 in the DLBCL cells was significantly lower than that in the GM12878 cell lines (Fig. 1A). In addition, the expression of GAS5 in the OCI-Ly3 and TMD8 cells was lower than that in the Raji cells; thus, the subcellular localization of GAS5 was observed in the OCI-Ly3, TMD8 and GM12878 cells. The corresponding results of fluorescence detection revealed that the expression level of GAS5 in the OCI-Ly3 and TMD8 cells was markedly lower than that in the GM12878 cells, and GAS5 was mainly located in the cytoplasm (Fig. 1B).

Overexpression of GAS5 regulates the proliferation, cell cycle progression and apoptosis of DLBCL cells. To evaluate the function of GAS5 in the proliferation, cell cycle progression and apoptosis of DLBCL cells, firstly, lentiviral vector containing the GAS5-encoding sequence was transfected into the OCI-Ly3 and TMD8 cells. The results revealed transfection with the GAS5 vector significantly promoted its expression level when compared with the empty vector group (Fig. 2A). The overexpression of GAS5 significantly decreased the viability and proliferation of the OCI-Ly3 and TMD8 cells when compared with the empty vector group (Fig. $2 \mathrm{~B}$ and C). In addition, GAS5 overexpression markedly suppressed the 
A

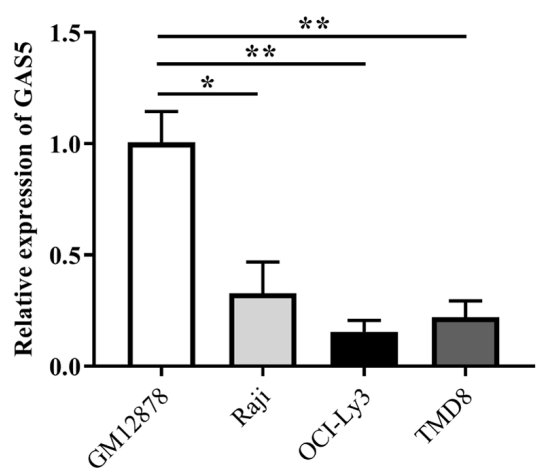

B

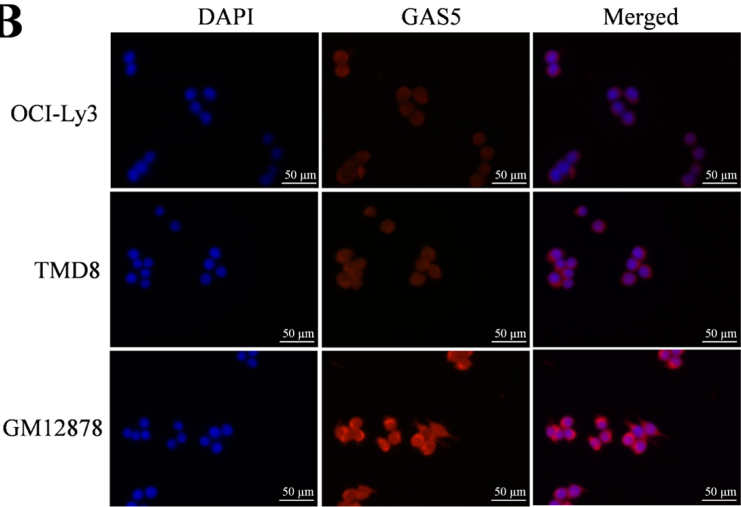

Figure 1. Detection of GAS5 expression. (A) The expression level of GAS5 in DLBCL cell lines (Raji, OCI-Ly3 and TMD8) and normal human B lymphocyte lines (GM12878) was detected using reverse transcription-quantitative PCR. (B) Location of GAS5 in the cytoplasm (red) and nucleus (blue) of OCI-Ly3, TMD8 and GM12878 cell lines was detected using fluorescence in situ hybridization. ${ }^{*} \mathrm{P}<0.05$ and ${ }^{* *} \mathrm{P}<0.01$. GAS5, growth arrest specific 5; DLBCL, diffuse large B-cell lymphoma.

A

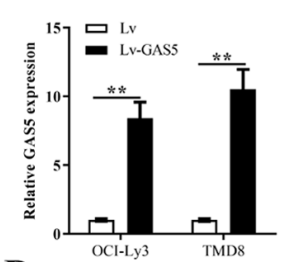

$\mathbf{D}$
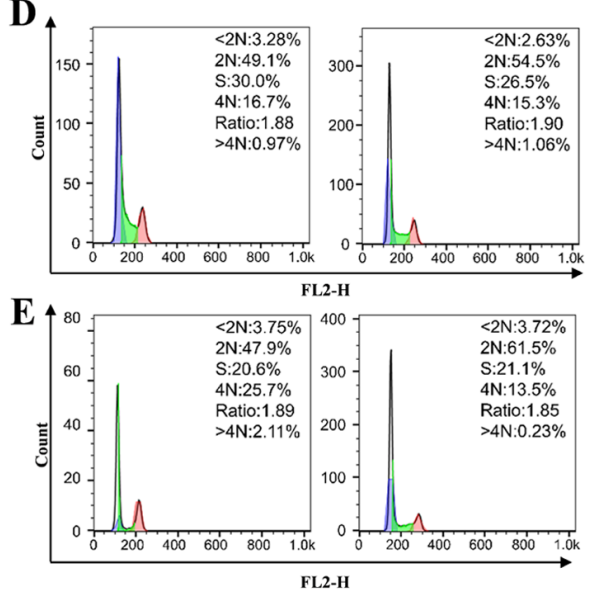

B
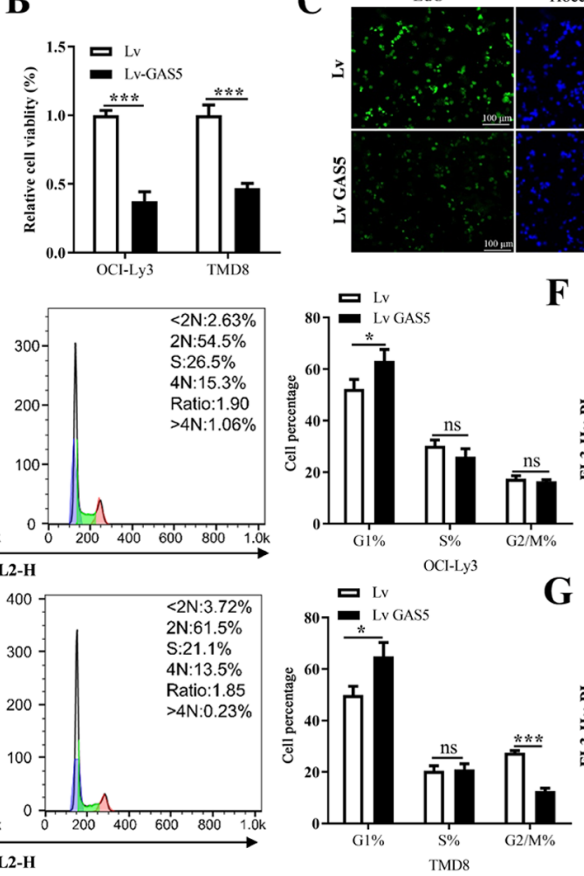

F

G
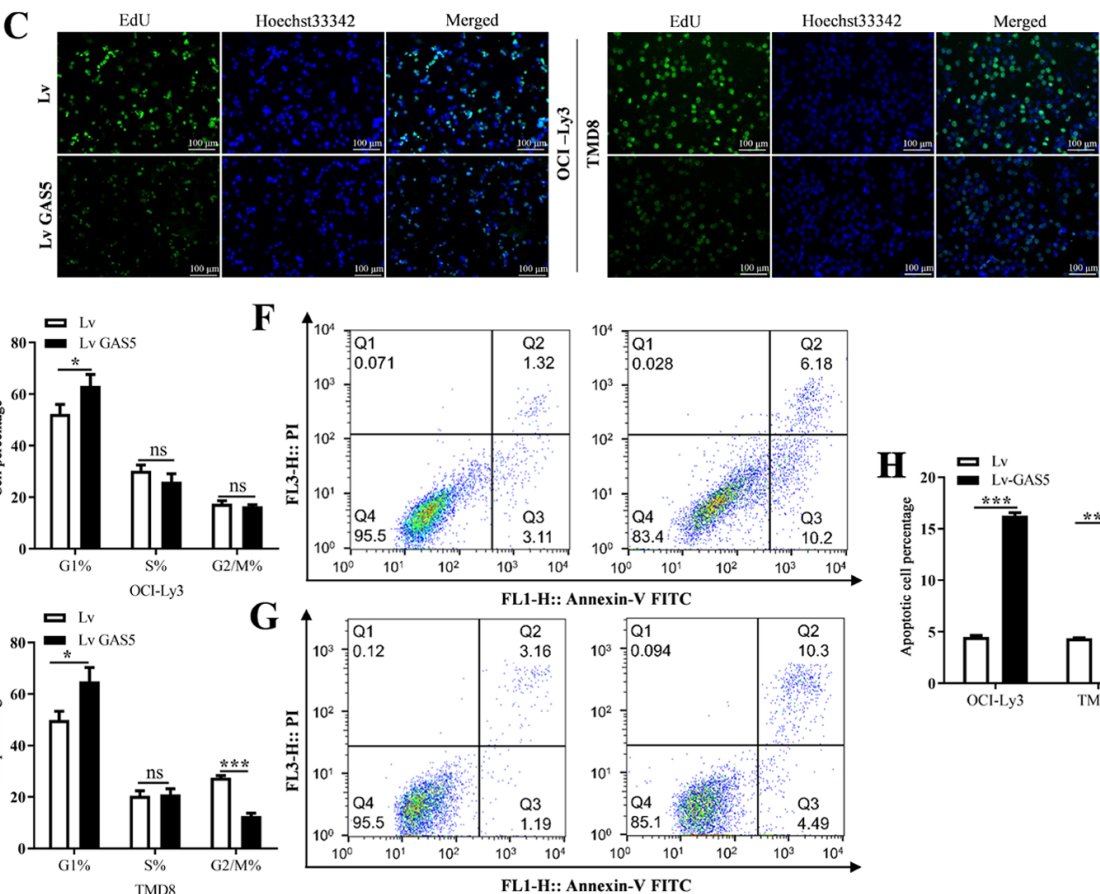

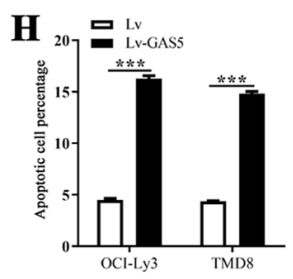

Figure 2. Detection of the effects of GAS5 on cell proliferation, cell cycle progression and apoptosis. (A) The transfection efficiency of the overexpression of GAS5 in OCI-Ly3 and TMD8 cell lines was detected using reverse transcription-quantitative PCR. (B and C) The viability and proliferative ability of OCI-Ly3 and TMD8 cells overexpressing GAS5 was detected using MTS and EdU assays. (D and E) The cell cycle of OCI-Ly3 and TMD8 overexpressing GAS5 was detected using flow cytometry. The apoptosis of (F) OCI-Ly3 and (G) TMD8 cells overexpressing GAS5 was detected using flow cytometry. (H) Quantitative analysis of cell apoptosis. ns, not significant; ${ }^{*} \mathrm{P}<0.05,{ }^{* *} \mathrm{P}<0.01$ and ${ }^{* * * *} \mathrm{P}<0.001$. GAS5, growth arrest specific 5; EdU, 5-ethynyl-2'-deoxyuridine.

cell cycle progression of the DLBCL cells, leading to G1 cycle arrest (Fig. 2D and E). Furthermore, the overexpression of GAS5 significantly increased the proportion of apoptotic cells when compared with the empty vector group (Fig. 2F, G and H).

GAS5 functions as a molecular sponge for miR-18a-5p. Three databases (starBase, DIANA and NPInter) were used to predict the target miRNAs of GAS5 (Table SI). As illustrated in Fig. 3A, five common miRNAs, including miR-135b-5p, miR-205-5p, miR-18a-5p, miR-23a-3p and miR-876-5p were predicted. Based on previous studies mentioned in the 'Introduction' $(24,25)$ and the database prediction results, miR-18a-5p was shown to be highly expressed in B-cell lymphoma, indicating that miR-18a-5p may be involved in the progression of DLBCL. Therefore, miR-18a-5p was selected for the follow-up mechanistic analysis. As was expected, the expression level of miR-18a-5p in the three DLBCL cell lines (Raji, OCI-ly3 and TMD8) was significantly higher than that in the GM12878 cell line (Fig. 3B). In addition, the binding site between GAS5 and miR-18a-5p was predicted, and the binding sequence of CGUGGAA was targeted and recognized by GAS5 (Fig. 3C). Moreover, the OCI-Ly3 cell line was used for mechanistic analysis, and therefore, the luciferase activity of the miR-18a-5p mimics and GAS5-WT co-transfection group was significantly decreased in the OCI-Ly3 cells when compared with that in the GAS5-MUT group. Additionally, no significant changes in luciferase activity 
A

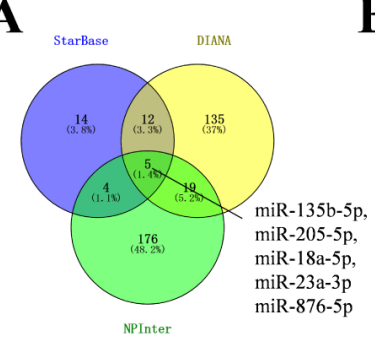

$\mathbf{E}$

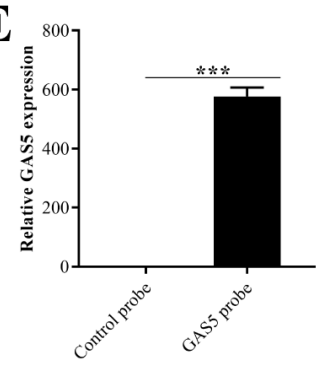

B
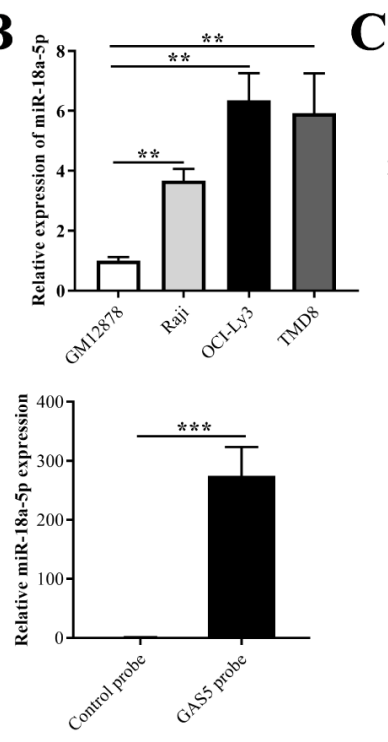

C
GAS5-WT 5, gu gug gCUCUgGaUAGCACCUU 3 ,

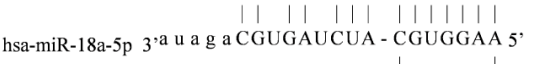

Gass-mut 5 'guguggCuCUgGauaAgaugcu 3 ,

D

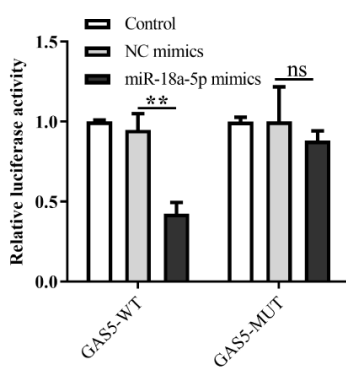

$\mathbf{F}$

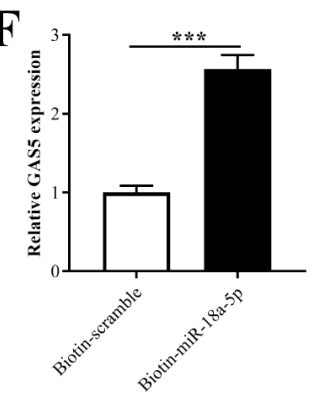

G

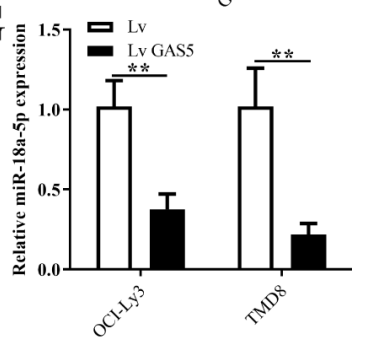

Figure 3. GAS5 functions as a molecular sponge for miR-18a-5p. (A) Venn diagram shows the targeted miRNAs that were predicted and screened using three online tools, including StarBase, DIANA and NPInter. (B) The expression level of miR-18a-5p in DLBCL cell lines (Raji, OCI-Ly3 and TMD8) and normal human B-lymphocyte lines (GM12878). (C) Schematic diagram illustrating the putative miR-18a-5p binding sites with the GAS5. (D) The relative luciferase activity in OCI-Ly3 cells co-transfected with luciferase reporter vectors of WT or MUT GAS5 and miR-18a-5p mimics. (E) The specificity of biotin-labeled GAS5 probe binding GAS5 and the pull-down of miR-18a-5p by GAS5 in total RNA from OCI-Ly3 cells. (F) Specificity binding of biotin-labeled miR-18a-5p and GAS5, and the pull-down of GAS5 by miR-18a-5p was detected using reverse transcription-quantitative PCR. (G) Relative expression level of miR-18a-5p in OCI-Ly3 and TMD8 cells overexpressing GAS5. ns, not significant; ${ }^{* *} \mathrm{P}<0.01$ and ${ }^{* * *} \mathrm{P}<0.001$. GAS5, growth arrest specific 5.

were observed in the control and NC mimic groups (Fig. 3D). The results of RT-qPCR revealed that the expression level of GAS5 increased with the combination of the biotin-labeled GAS5 probe and GAS5, indicating the specific binding between the probe and GAS5. In addition, RT-qPCR revealed that the probe specifically bound by GAS5 'pulled down' a greater amount of miR-18a-5p in the total RNA of OCI-Ly3 cells than in the control group (Fig. 3E). Furthermore, the RNA pulled down by biotin-labeled miR-18a-5p was enriched by magnetic beads by RNA Pull-down. The corresponding RT-qPCR results confirmed that miR-18a-5p could specifically bind to GAS5 compared with the biotin-scramble (Fig. 3F). Furthermore, the overexpression of GAS5 decreased the relative expression level of miR-18a-5p in the OCI-Ly3 and TMD8 cells when compared with the control group (Fig. 3G).

GAS5 functions as a ceRNA by sponging miR-18a-5p. Four databases (TargetScan, PicTar, TarBase and microT_CDS) were used to predict the target genes of miR-18a-5p (Table SII). As illustrated in Fig. 4A, 22 common genes (ADD3, BTG3, CREBL2, CRIM1, CTGF, GCLC, HIF1A, HSF2, IRF2, NR3C1, PDE4D, PTGFRN, PURB, RUNX1, SATB1, SH3BP4, SIM2, SOCS5, XYLT2, YPEL5, ZBTB4 and ZNF367) were predicted. Based on the previous studies mentioned in the 'Introduction' (27-29) and the database predictions results, RUNX1 was selected for the follow-up mechanistic analysis. As was expected, the expression level of RUNX1 was significantly lower in the three DLBCL cell lines (Raji, OCI-ly3 and TMD8), whereas it was higher in the GM12878 cells (Fig. 4B). RUNX1 was identified as a novel target of miR-18a-5p, and the binding site of GCACCUU was then predicted (Fig. 4C). In addition, the results of luciferase reporter assay revealed a significant reduction in the luciferase activity following co-transfection with the miR-18a-5p mimic and RUNX1-WT when compared to co-transfection with the control or NC mimics and RUNX1-WT groups, while no significant difference was observed in the RUNX1-MUT transfection groups (Fig. 4D). To further confirm the interaction between miR-18a-5p and RUNX1, miR-18a-5p mimics and inhibitor were transfected into the OCI-ly 3 and TMD8 cells. The results revealed that miR-18a-5p expression was increased and suppressed following transfection with miR-18a-5p mimics and inhibitor, respectively (Fig. 4E). Moreover, it was found that the overexpression or inhibition of miR-18a-5p significantly decreased or increased the mRNA and protein expression of RUNX1, respectively (Fig. 5A and B). In addition, the results of RT-qPCR and western blot analysis revealed that the overexpression of GAS5 enhanced the mRNA and protein expression of RUNX1 (Fig. 5C and D). Furthermore, compared with the OCI-ly3 and TMD8 cells transfected with miR-18a-5p mimics or GAS5 overexpression vector alone, those co-transfected with miR-18a-5p mimics and GAS5 overexpression vector exhibited an increased or decreased RUNX1 expression, respectively (Fig. 5E and F).

Knockdown of RUNX1 reverses the GAS5-induced inhibition of the proliferation of DLBCL cells. To explore whether RUNX1 was involved in the proliferation, cell cycle progression and apoptosis of DLBCL cells regulated by GAS5, the expression level of RUNX1 was examined following the knockdown of RUNX1. The results revealed that the expression of RUNX1 was significantly decreased compared with the control group (Fig. 6A). Furthermore, the knockdown of RUNX1 decreased its protein expression level (Fig. 6B). The results of rescue experiments then revealed that co-transfection of the OCI-LY3 and TMD8 cells with GAS5 overexpression vector and si-RUNX1 reversed the inhibitory effects of GAS5 
A

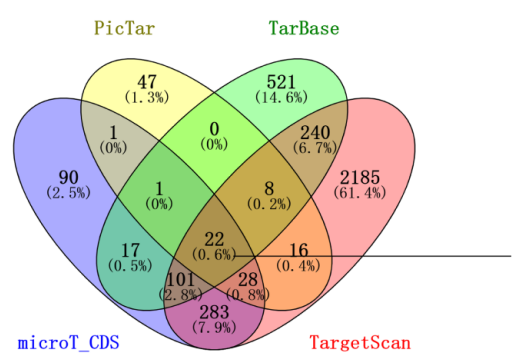

ADD3, BTG3, CREBL2, CRIMI, CTGF, GCLC, HIF1A, HSF2, IRF2, NR3C1, PDE4D, PTGFRN, PURB, RUNX1, SATB1, SH3BP4, SIM2, SOCS5, XYLT2, YPEL5, ZBTB4, ZNF367
B

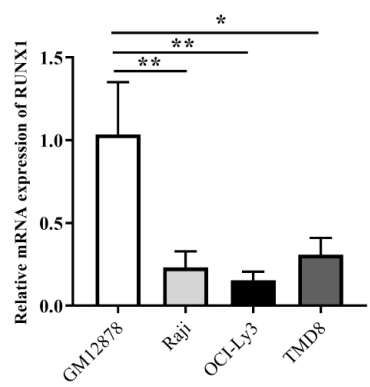

E

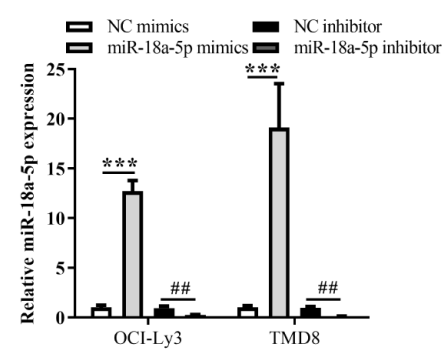

Figure 4. RUNX1 is predicted as a direct target of miR-18a-5p. (A) The Venn diagram shows the targeted mRNAs that were predicted and screened by four online tools including TargetScan, PicTar, Tarbase and microT_CDS. (B) The expression level of RUNX1 in DLBCL cell lines (Raji, OCI-Ly3 and TMD8) and a normal human B-lymphocyte cell line (GM12878). (C) Schematic diagram illustrating the predicted miR-18a-5p binding sites with the 3'UTR of RUNX1. (D) The relative luciferase activity in OCI-Ly3 cells co-transfected with luciferase reporter vectors of RUNX1-WT or RUNX1-MUT and miR-18a-5p mimics (E) The transfection efficiency of miR-18a-5p mimics/inhibitor in OCI-Ly3 and TMD8 cell lines. ${ }^{*} \mathrm{P}<0.05,{ }^{* *} \mathrm{P}<0.01$ and ${ }^{* * * *} \mathrm{P}<0.001$; ${ }^{\# *} \mathrm{P}<0.01$. ns, not significant; RT-qPCR, reverse transcription-quantitative PCR; RUNX1, Runt-related transcription factor 1.

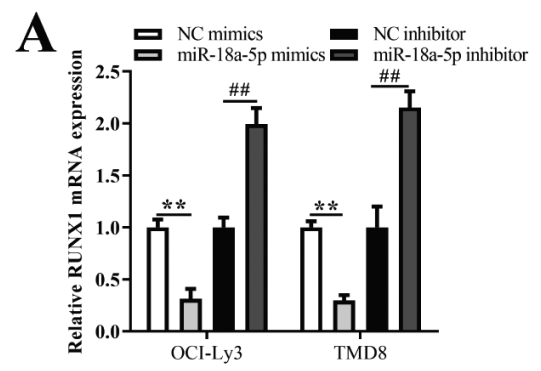

C

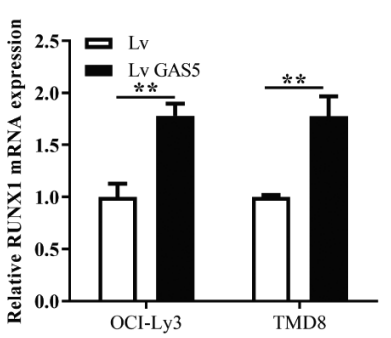

$\mathbf{E}$

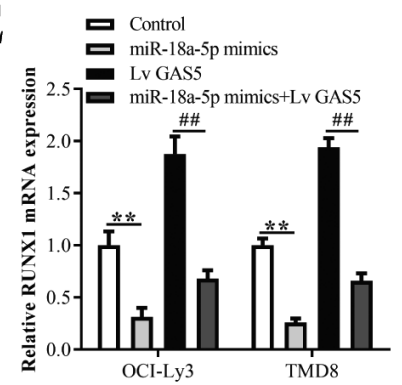

B

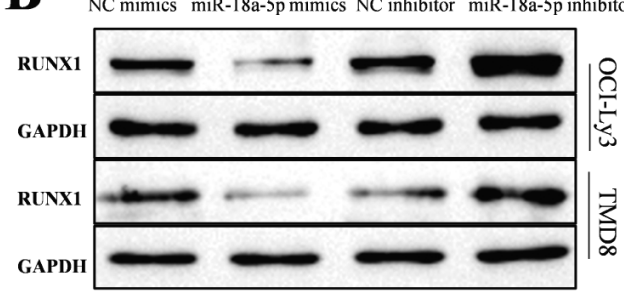

D

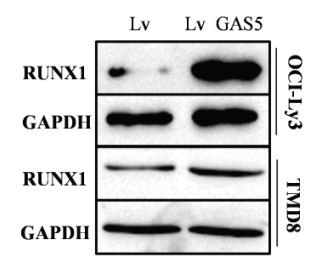

F

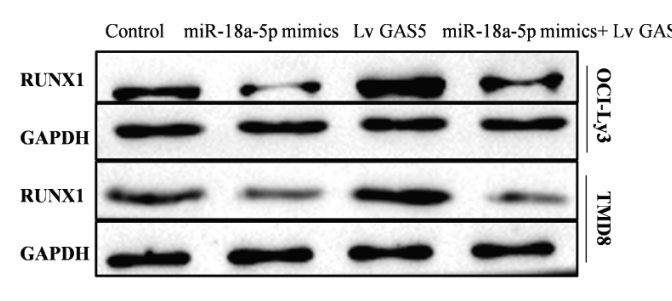

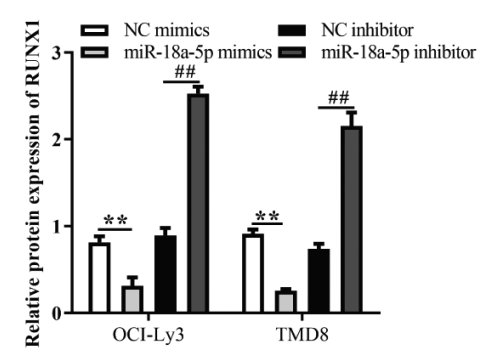
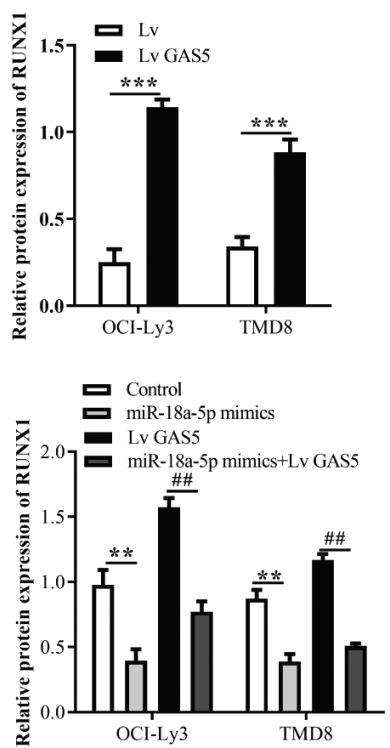

Figure 5. RUNX1 is regulated by GAS5 and miR-18a-5p. (A and B) RUNX1 expression was detected using RT-qPCR and western blot analysis following after transfection with miR-18a-5p mimics/inhibitor into OCI-Ly3 and TMD8 cells. (C and D) The mRNA and protein expression of RUNX1 in OCI-Ly3 and TMD8 cells overexpressing GAS5 was detected using RT-qPCR and western blot analysis. (E and F) The mRNA and protein expression of RUNX1 in OCI-Ly3 and TMD8 cells transfected with GAS5 overexpression vector and/or miR-18a-5p mimics was detected using RT-qPCR and western blot analysis. ns, not significant; ${ }^{* *} \mathrm{P}<0.01$ and ${ }^{* * *} \mathrm{P}<0.001 ;{ }^{\# \#} \mathrm{P}<0.01$. RT-qPCR, reverse transcription-quantitative PCR; GAS5, growth arrest specific 5; RUNX1, Runt-related transcription factor 1. 


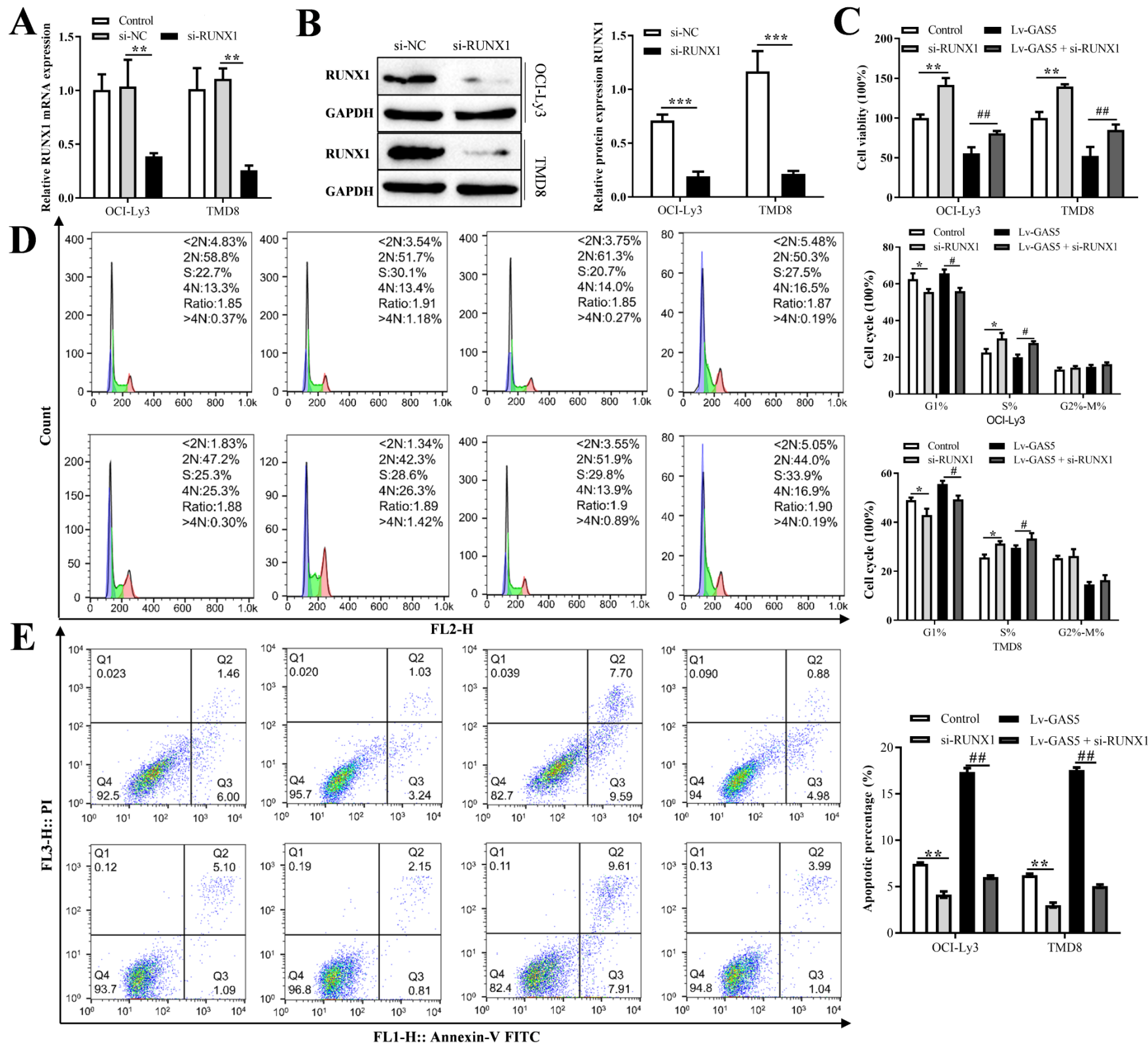

Figure 6. Knockdown of RUNX1 reverses the effects of GAS5 overexpression on the proliferation, cell cycle progression and apoptosis of TMD8 and OCI-Ly3 cells. (A and B) The transfection efficiency of si-RUNX1 in OCI-Ly3 and TMD8 cell lines was detected using reverse transcription-quantitative PCR. (C) Viability of OCI-Ly3 and TMD8 cells was detected using MTS assay following transfection with GAS5 overexpression vector and or si-RUNX1. (D) The cell cycle was detected using flow cytometry in OCI-Ly3 and TMD8 cells transfected with GAS5 overexpression vector and or si-RUNX1. (E) Apoptosis of OCI-Ly3 and TMD8 cells was detected using flow cytometry following transfection with GAS5 overexpression vector and or si-RUNX1. ${ }^{*} \mathrm{P}<0.05$, ${ }^{* *} \mathrm{P}<0.01$ and ${ }^{* * *} \mathrm{P}<0.001 ;{ }^{\#} \mathrm{P}<0.05$ and ${ }^{\# \#} \mathrm{P}<0.01$. GAS5, growth arrest specific 5; RUNX1, Runt-related transcription factor 1.

overexpression on the proliferation of OCI-LY3 and TMD8 cells; the cell cycle arrest in the G1 phase and the promotion of cell apoptosis (Fig. 6C-E).

RUNX1 promotes the expression of BAX and binds to its promoter. JASPAR database query results revealed that a potential binding site ACTTGAGGT of RUNX1 was found within the sequence 2000 upstream of the promoter region of BAX. The results of RT-qPCR and western blot analysis revealed that the knockdown of RUNX1 decreased the mRNA and protein expression of BAX (Fig. 7A and B). Moreover, luciferase activity was used to evaluate the binding of RUNX1 to the BAX promoter. As was expected, RUNX1-WT increased the activity of the BAX promoter (Fig. 7C). Furthermore, immunoprecipitation assay of the protein-DNA complexes was performed, and the results of ChIP-qPCR assay confirmed that RUNX1 protein enriched the binding site and enhanced the interaction within the BAX promoter in OCI-Ly3 cells, but not binding to the control region (Fig. 7D). In summary, lncRNA GAS5 promotes the cell cycle arrest, suppresses the proliferation and promotes the apoptosis of OCI-Ly3 and TMD8 cells by sponging miR-18a-5p and regulating the expression of RUNX1 (Fig. 8).

\section{Discussion}

The present study first established the interaction among GAS5, miR-18a-5p and RUNX1 in DLBCL, and concluded 

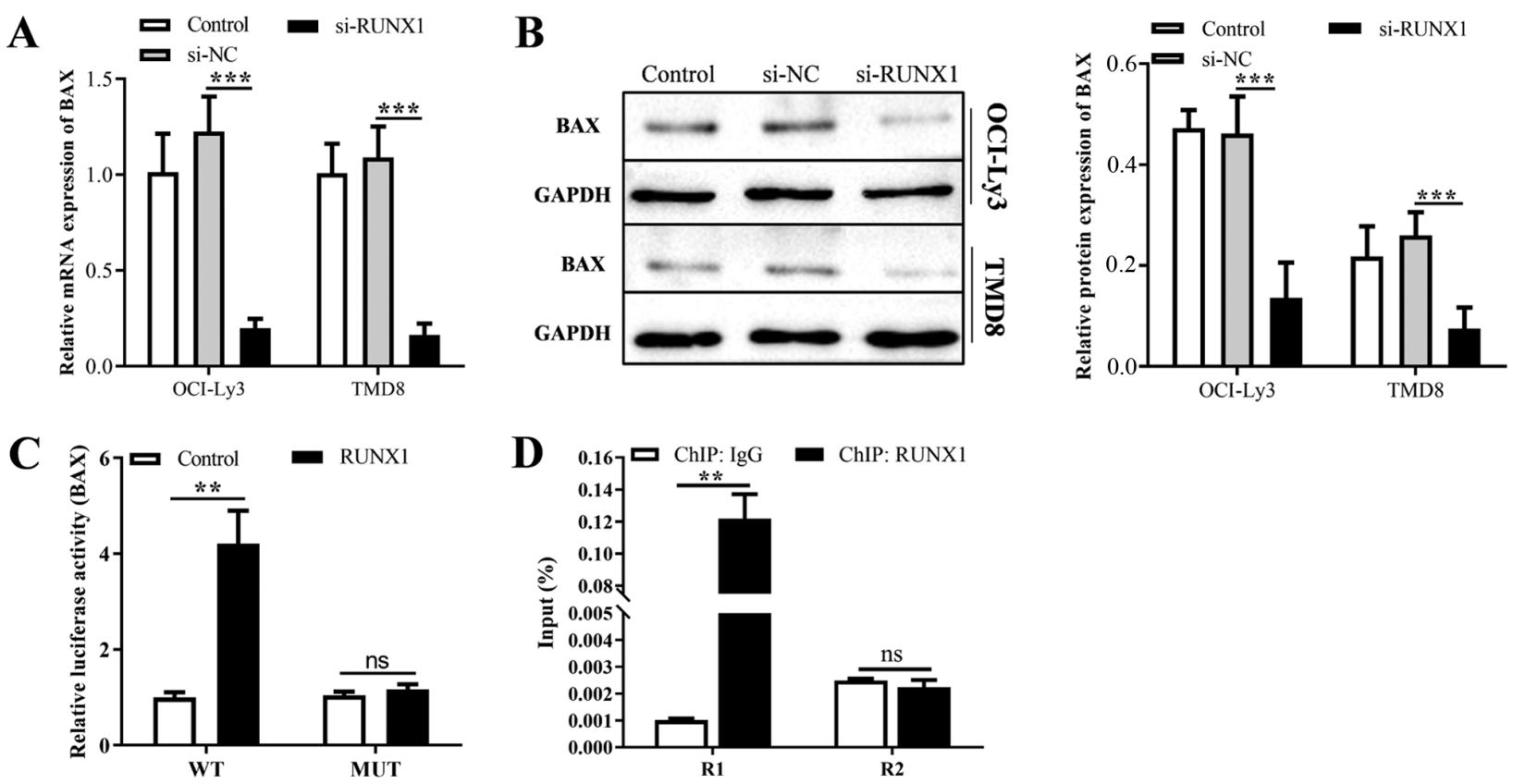

Figure 7. Detection of the binding of RUNX1 and BAX promoter. (A and B) BAX expression in TMD8 and OCI-Ly3 cells transfected with si-RUNX1 was detected using reverse transcription-quantitative PCR and western blot analysis. (C) Effect of RUNX1 on BAX activity in OCI-Ly3 cells was detected using luciferase activity. (D) A ChIP-qPCR analysis of the BAX promoter sequence with the addition of antibodies, including normal IgG (negative control) and anti-RUNX1 (positive control) in OCI-Ly3 cells. R1, binding site on the BAX promoter region; R2, control region, ns, not significant; ${ }^{* *} \mathrm{P}<0.01$ and ${ }^{* * *} \mathrm{P}<0.001$. GAS5, growth arrest specific 5; RUNX1, Runt-related transcription factor 1.

that GAS5 inhibited the proliferation and G1 cycle progression, whereas it promoted the apoptosis of DLBCL cells by functioning as a ceRNA to sponge miR-18a-5p and modulate RUNX1 expression. These findings may provide potential novel therapeutic targets for the treatment of DLBCL (Fig. 7).

The abnormal expression of lncRNAs has been identified as a main factor involved in the progression of DLBCL. The association between IncRNAs and cell proliferation, and the apoptosis of DLBCL has been previously demonstrated; for example, IncRNA SNHG16 (31), IncRNA OR3A4 (32), lncRNA AFAP1-AS1 (33) have been shown to play a role in B-cell lymphoma. A previous study demonstrated that a low GAS5 level was involved in the development of DLBCL and was associated with a poor prognosis; moreover, the results of protein-protein interaction network (PPI) network analysis revealed that GAS5 negatively regulated the cell cycle, apoptosis, differentiation, autophagy and other cell functions (18). This finding is in agreement with findings of previous studies demonstrating that GAS5 plays the role of a tumor suppressor gene by inhibiting the proliferation and promoting the apoptosis of tumor cells in glioma and ovarian cancers $(20,34,35)$. Indeed, the overexpression of GAS5 has been shown to reduce the expression of the anti-apoptotic protein, $\mathrm{Bcl}-2$, to promote the apoptosis of bladder cancer and cervical cancer cells (36). However, to date, to the best of our knowledge, there is no direct evidence to verify the association between GAS5, and the proliferation and apoptosis of DLBCL cells. In the present study, it was also found that GAS5 was expressed at low levels in DLBCL cells, indicating the role of GAS5 in this disease. Functionally, GAS5 reduced the proliferation of DLBCL cells and enhanced cell apoptosis. In addition, the results revealed that GAS5 induced G1 cell cycle arrest, which was also similar with the findings of a previous study (37). However, another study demonstrated that GAS5 was highly expressed in DLBCL, whereas its expression was low in follicular lymphoma (FL) blood samples by analyzing a cohort of previously published datasets from the Gene Expression Omnibus (GEO; accession no. GSE53820), and the lncRNA-mRNA co-expression network analysis results revealed that GAS5 was associated with cell anti-apoptosis, cell cycle and other functions (38). This may be due to the fact that only six DLBCL blood samples were included in that study. Moreover, differential analysis and multiple tests on all genes in the DLBCL and FL samples were performed using the online analysis tool, GEO2R (https://www.ncbi.nlm.nih. gov/geo/geo $2 \mathrm{r} /$ ); the results revealed that the expression level of GAS5 in DLBCL and FL was no longer significant (the adjusted P-value was 0.53) (Table SIII).

Mechanistically, the findings of the present study validated that GAS5 functioned as a miRNA sponge in DLBCL. As previously reported, GAS5 may participate in the specific DLBCL process as a key regulator of the ceRNA network (38). The interaction between lncRNAs and miRNAs involves the regulation of target mRNAs, and IncRNAs may sponge and reduce miRNA expression to compete for the regulatory effects of miRNAs on target gene mRNAs, also known as the ceRNA mechanism. Previous studies have revealed that GAS5 competes with miRNAs to regulate mRNA expression as a ceRNA $(20,39)$. Notably, the present study predicted that miR-18a-5p was the potential target gene of by GAS5 through three online prediction database and validated that miR-18a-5p was directly targeted by GAS5 in OCI-Ly 3 cells by using luciferase reporter and RNA pull-down assays. Furthermore, the present study demonstrated that miR-18a-5p expression was markedly upregulated in DLBCL cell lines, and exhibited a strong affinity with GAS5, indicating that GAS5 may 


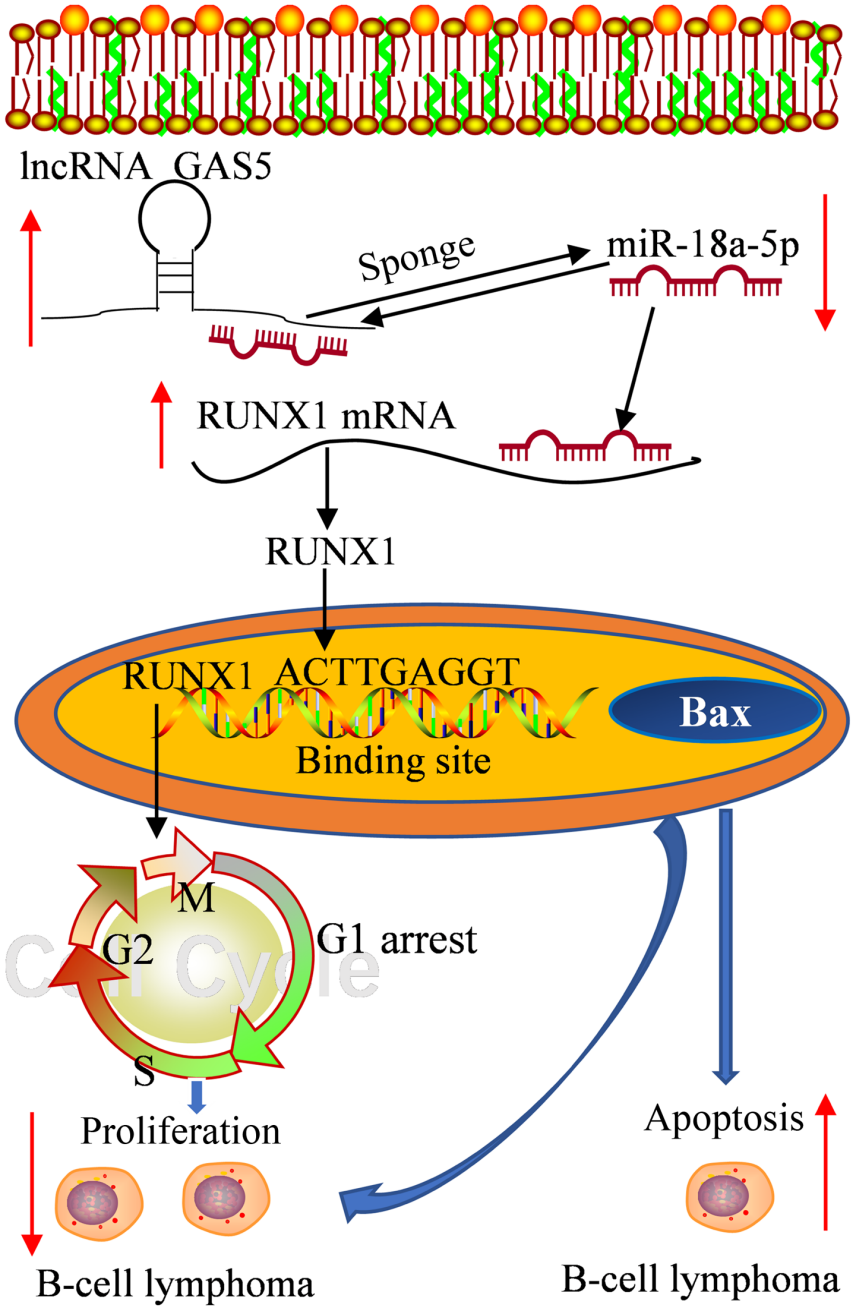

Figure 8. Schematic diagram of the mechanisms through which lncRNA GAS5/miR-18a-5p/RUNX1 mediates DLBCL cell proliferation and apoptosis. 1ncRNA GAS5 attenuates the expression of miR-18a-5p, whereas it promotes that of RUNX1; miR-18a-5p attenuates the expression of RUNX1 and interferes with the expression of RUNX1 by GAS5. On the whole, lncRNA GAS5 promotes the expression of RUNX1 by sponging miR-18a-5p, which in turn promotes the cell cycle G1 phase arrest, suppresses proliferation and promotes the apoptosis of OCI-Ly3 and TMD8 cells. In addition, RUNX1 binds to the promoter region of BAX, and thus may play a role in the apoptosis and proliferation of DLBCL cells. lncRNA, long non-coding RNA; GAS5, growth arrest specific 5; RUNX1, Runt-related transcription factor 1; DLBCL, diffuse large B-cell lymphoma.

function in DLBCL mainly through miR-18a-5p. Similarly, it has been proven in previous studies that miR-18a-5p plays a key carcinogenic role in several tumors and is negatively regulated by GAS5 in glioma (20) and prostate cancer cells (26). In prostate cancer, $\alpha$-solanine is involved in the regulation of tumor inhibition of miR-18a-5p; however, those studies did not include RUNX1.

Of note, the present study demonstrated that RUNX1 was expressed at low levels in DLBCL and was targeted by GAS5 and miR-18a-5p, suggesting an involvement of RUNX1 in DLBCL cells. Functionally, GAS5 decreased the proliferation of DLBCL cells, and induced G1 phase arrest and cell apoptosis. However, in rescue experiments, co-transfection with GAS5 overexpression and si-RUNX1 reversed the effects of GAS5 on the proliferation, cell cycle progression and apoptosis of DLBCL cells. Similar results have also been observed in other tumors. For example, RUNX1 has been shown to markedly inhibit the IncRNA NEF-induced proliferation of gastric cancer cells (40) and the IncRNA CASC2-induced proliferation of malignant melanoma cells (41). However, some studies have indicated that RUNX1 can promote tumor development; for example, RUNX1 has been shown to reverse the anti-proliferative and pro-apoptotic effects on glioma cells following the knockdown of lncRNA HCP5 (42), or to enhance the IncRNA RNCR3-induced progression of colorectal cancer (43). In addition, previous studies have also revealed that RUNX1 regulates G1 to $\mathrm{S}$ cell cycle progression in adult hematopoietic stem cells (44), and the knockdown of RUNX1 has been shown to lead to a decrease in cell proliferation and to G1 cell cycle arrest in epithelial ovarian carcinoma (45); these findings were similar to those of the present study. The aforementioned results suggest that GAS5 and RUNX1 may be potential therapeutic targets for DLBCL.

Furthermore, RUNX1 is a transcription factor that is well known in the development of cancers for its dual role in the transcription of specific genes. For example, RUNX1 binds to the promoter region of the glioma oncogene astrocyte elevated gene-1 (AEG-1), enhances the activity of the AEG-1, and then induces the proliferation of glioma cells (42). Of note, the results of RT-qPCR, and luciferase activity and ChIP assays in the present study proved that RUNX1 increased the activity of BAX and enhanced BAX expression. It has been reported that $\mathrm{BAX}$ is a key regulatory protein related to apoptosis and the proliferation of DLBCL cells (46). A previous study also identified that RUNX1 enhanced the transcriptional activity of BAX, and si-RUNX1 was shown to play an anti-apoptotic role in human colon carcinoma (47). Based on these studies, RUNX1 may be a key factor in the BAX-mediated cell proliferation and apoptosis of DLBCL, which may further confirm the tumor suppressor effect of RUNX1 in DLBCL in the present study.

In conclusion, the present study provides evidence that lncRNA GAS5 plays an anti-tumor and anti-proliferative role in DLBCL cells. In addition, the potential mechanism identified was that it inhibited the proliferation and G1 cycle progression, and promoted the apoptosis of DLBCL cells by functioning as a ceRNA to regulate RUNX1. These findings provide potential novel therapeutic targets for DLBCL.

\section{Acknowledgements}

Not applicable.

\section{Funding}

The present study was supported by the Medical Research Project of Xi'an Science and Technology Bureau (grant no. 2019114613YX001SF037-4), the Key Project of Natural Science Foundation of Shaanxi Provincial Science and Technology Department (grant no. 2016JZ030) and the Natural Fund General Project of Science and Technology Department of Shaanxi Province (Youth Project; grant no. 2018JQ8071).

\section{Availability of data and materials}

All data generated or analyzed during this study are included in this published article. 


\section{Authors' contributions}

YM and YJ performed the experiments and collected the data, and confirmed the authenticity of all the raw data. YM was a major contributor to the writing of the manuscript. XC and MQ were responsible for data analysis and visualization. WZ and YW conceived and designed the study, and they were major contributors in critically revising the manuscript. All authors have read and approved the final manuscript.

\section{Ethics approval and consent to participate}

Not applicable.

\section{Patient consent for publication}

Not applicable.

\section{Competing interests}

The authors declare that they have no competing interests.

\section{References}

1. Offner F, Samoilova O, Osmanov E, Eom HS, Topp MS Raposo J, Pavlov V, Ricci D, Chaturvedi S, Zhu E, et al: Frontline rituximab, cyclophosphamide, doxorubicin, and prednisone with bortezomib (VR-CAP) or vincristine (R-CHOP) for non-GCB DLBCL. Blood 126: 1893-1901, 2015.

2. Alizadeh AA, Eisen MB, Davis RE, Ma C, Lossos IS, Rosenwald A, Boldrick JC, Sabet H, Tran T, Yu X, et al: Distinct types of diffuse large B-cell lymphoma identified by gene expression profiling. Nature 403: 503-511, 2000

3. Lee WJ, Won KH, Won CH, Chang SE, Choi JH, Moon KC, Park CS, Huh J, Suh C and Lee MW: Secondary cutaneous diffuse large B-cell lymphoma has a higher international prognostic index score and worse prognosis than diffuse large B-cell lymphoma, leg type. Acta Derm Venereol 96: 245-250, 2016.

4. Castillo JJ, Winer ES and Olszewski AJ: Sites of extranodal involvement are prognostic in patients with diffuse large B-cell lymphoma in the rituximab era: An analysis of the Surveillance, Epidemiology and End Results database. Am J Hematol 89: 310-314, 2014

5. Li S, Young KH and Medeiros LJ: Diffuse large B-cell lymphoma. Pathology 50: 74-87, 2018.

6. Wang QM, Lian GY, Song Y, Huang YF and Gong Y: LncRNA MALAT1 promotes tumorigenesis and immune escape of diffuse large B cell lymphoma by sponging miR-195. Life Sci 231: 116335,2019

7. Shi X, Cui Z, Liu X, Wu S, Wu Y, Fang F and Zhao H: LncRNA FIRRE is activated by MYC and promotes the development of diffuse large B-cell lymphoma via Wnt/B-catenin signaling pathway. Biochem Biophys Res Commun 510: 594-600, 2019.

8. Qian CS, Li LJ, Huang HW, Yang HF and Wu DP: MYC-regulated lncRNA NEAT1 promotes B cell proliferation and lymphomagenesis via the miR-34b-5p-GLI1 pathway in diffuse large B-cell lymphoma. Cancer Cell Int 20: 87, 2020.

9. Zhao CC, Jiao Y, Zhang YY, Ning J, Zhang YR, Xu J, Wei W and Kang-Sheng G: Lnc SMAD5-AS1 as ceRNA inhibit proliferation of diffuse large B cell lymphoma via Wnt/ $\beta$-catenin pathway by sponging miR-135b-5p to elevate expression of APC. Cell Death Dis 10: 252, 2019.

10. Wang Y, Zhang M, Xu H, Wang Y, Li Z, Chang Y, Wang X, Fu X, Zhou Z, Yang S, et al: Discovery and validation of the tumor-suppressive function of long noncoding RNA PANDA in human diffuse large B-cell lymphoma through the inactivation of MAPK/ERK signaling pathway. Oncotarget 8: 72182-72196, 2017

11. Jiang L, Zhao XH, Mao YL, Wang JF, Zheng HJ and You QS: Long non-coding RNA RP11-468E2.5 curtails colorectal cancer cell proliferation and stimulates apoptosis via the JAK/STAT signaling pathway by targeting STAT5 and STAT6. J Exp Clin Cancer Res 38: 465, 2019.
12. Li J, Wang Y, Zhang CG, Xiao H-J, Xiao HJ, Hu J-M, Hou JM and He JD: Effect of long non-coding RNA Gas5 on proliferation, migration, invasion and apoptosis of colorectal cancer HT-29 cell line. Cancer Cell Int 18: 4, 2018.

13. Liu Y, Yin L, Chen C, Zhang X and Wang S: Long non-coding RNA GAS5 inhibits migration and invasion in gastric cancer via interacting with $\mathrm{p} 53$ protein. Dig Liver Dis 52: 331-338, 2020

14. Huang W, Shi Y, Han B, Wang Q, Zhang B, Qi C and Liu F: LncRNA GAS5-AS1 inhibits glioma proliferation, migration, and invasion via miR-106b-5p/TUSC2 axis. Hum Cell 33: 416-426, 2020

15. Zheng S, Li M, Miao K and Xu H: lncRNA GAS5-promoted apoptosis in triple-negative breast cancer by targeting miR-378a-5p/ SUFU signaling. J Cell Biochem 121: 2225-2235, 2020.

16. Nakamura Y, Takahashi N, Kakegawa E, Yoshida K, Ito Y, Kayano H, Niitsu N, Jinnai I and Bessho M: The GAS5 (growth arrest-specific transcript 5) gene fuses to BCL6 as a result of $\mathrm{t}(1 ; 3)(\mathrm{q} 25 ; \mathrm{q} 27)$ in a patient with B-cell lymphoma. Cancer Genet Cytogenet 182: 144-149, 2008.

17. Dousti F, Shahrisa A, Ansari H, Hajjari M, Tahmasebi Birgani Y, Mohammadiasl $\mathbf{J}$ and Tahmasebi Birgani M: Long non-coding RNAs expression levels in diffuse large B-cell lymphoma: An in silico analysis. Pathol Res Pract 214: 1462-1466, 2018.

18. Senousy MA, El-Abd AM, Abdel-Malek RR and Rizk SM: Circulating long non-coding RNAs HOTAIR, Linc-p21, GAS5 and XIST expression profiles in diffuse large B-cell lymphoma: Association with R-CHOP responsiveness. Sci Rep 11: 2095, 2021.

19. Luengo-Gil G, García-Martínez E, Chaves-Benito A, Conesa-Zamora P, Navarro-Manzano E, González-Billalabeitia E, García-Garre E, Martínez-Carrasco A, Vicente V and Ayala de la Peña F: Clinical and biological impact of miR-18a expression in breast cancer after neoadjuvant chemotherapy. Cell Oncol (Dordr) 42: 627-644, 2019.

20. Liu Q, Yu W, Zhu S, Cheng K, Xu H, Lv Y, Long X, Ma L, Huang J, Sun S, et al: Long noncoding RNA GAS5 regulates the proliferation, migration, and invasion of glioma cells by negatively regulating miR-18a-5p. J Cell Physiol 234: 757-768, 2018.

21. Liang C, Zhang X, Wang HM, Liu XM, Zhang XJ, Zheng B, Qian GR and Ma ZL: MicroRNA-18a-5p functions as an oncogene by directly targeting IRF2 in lung cancer. Cell Death Dis 8: e2764, 2017.

22. Miao WJ, Yuan DJ, Zhang GZ, Liu Q, Ma HM and Jin QQ: lncRNA CASC $2 / \mathrm{miR} 18$ a $5 \mathrm{p}$ axis regulates the malignant potential of nasopharyngeal carcinoma by targeting RBBP8. Oncol Rep 41: 1797-1806, 2019.

23. Liang B, Zhou C, Cui S, Lu H, Xu R, Xue D, Zou S and He X: Upregulation of miR-18a-5p promotes the proliferation of prostate cancer via inhibiting the expression of SLC40A1. Pathol Res Pract 224: 153448, 2021.

24. Lim EL, Trinh DL, Scott DW, Chu A, Krzywinski M, Zhao Y, Robertson AG, Mungall AJ, Schein J, Boyle M, et al: Comprehensive miRNA sequence analysis reveals survival differences in diffuse large B-cell lymphoma patients. Genome Biol 16: 18, 2015.

25. He L, Thomson JM, Hemann MT, Hernando-Monge E, Mu D, Goodson S, Powers S, Cordon-Cardo C, Lowe SW, Hannon GJ, et al: A microRNA polycistron as a potential human oncogene. Nature 435: 828-833, 2005

26. Yang J, Hao T, Sun J, Wei $P$ and Zhang $H$ : Long noncoding RNA GAS5 modulates $\alpha$-Solanine-induced radiosensitivity by negatively regulating miR-18a in human prostate cancer cells. Biomed Pharmacother 112: 108656, 2019.

27. Sood R, Kamikubo Y and Liu P: Role of RUNX1 in hematological malignancies. Blood 129: 2070-2082, 2017.

28. Seo W, Ikawa T, Kawamoto H and Taniuchi I: Runx1-Cbf $\beta$ facilitates early B lymphocyte development by regulating expression of Ebf1. J Exp Med 209: 1255-1262, 2012

29. Miao YS, Zhao YY, Zhao LN, Wang P, Liu YH, Ma J and Xue YX: MiR-18a increased the permeability of BTB via RUNX1 mediated down-regulation of ZO-1, occludin and claudin-5. Cell Signal 27: 156-167, 2015.

30. Livak KJ and Schmittgen TD: Analysis of relative gene expression data using real-time quantitative PCR and the 2(-Delta Delta C(T)) method. Methods 25: 402-408, 2001.

31. Zhu Q, Li Y, Guo Y, Hu L, Xiao Z, Liu X, Wang J, Xu Q and Tong X: Long non-coding RNA SNHG16 promotes proliferation and inhibits apoptosis of diffuse large B-cell lymphoma cells by targeting miR-497-5p/PIM1 axis. J Cell Mol Med 23: 7395-7405, 2019. 
32. Meng H, Zhao B and Wang Y: FOXM1-induced upregulation of lncRNA OR3A4 promotes the progression of diffuse large B-cell lymphoma via Wnt/ $\beta$-catenin signaling pathway. Exp Mol Pathol 115: 104451, 2020.

33. Gao H, Sun Y, Chen J, Jin H and Yang W: Long non-coding RNA AFAP1-AS1 promotes cell growth and inhibits apoptosis by binding to specific proteins in germinal center B-cell-like diffuse large B-cell lymphoma. Am J Transl Res 12: 8225-8246, 2020.

34. Li J, Yang C, Li Y, Chen A, Li L and You Z: LncRNA GAS5 suppresses ovarian cancer by inducing inflammasome formation. Biosci Rep 38: 38, 2018.

35. Li G, Cai Y, Wang C, Huang M and Chen J: LncRNA GAS5 regulates the proliferation, migration, invasion and apoptosis of brain glioma cells through targeting GSTM3 expression. The effect of LncRNA GAS5 on glioma cells. J Neurooncol 143: 525-536, 2019.

36. Zhao W, Shan B, He D, Cheng Y, Li B, Zhang C and Duan C: Recent progress in characterizing long noncoding RNAs in cancer drug resistance. J Cancer 10: 6693-6702, 2019.

37. Yang Y, Shen Z, Yan Y, Wang B, Zhang J, Shen C, Li T, Ye C, Gao Z, Peng G, et al: Long non-coding RNA GAS5 inhibits cell proliferation, induces G0/G1 arrest and apoptosis, and functions as a prognostic marker in colorectal cancer. Oncol Lett 13: 3151-3158, 2017.

38. Tian L, He Y, Zhang H, Wu Z, Li D and Zheng C: Comprehensive analysis of differentially expressed profiles of lncRNAs and mRNAs reveals ceRNA networks in the transformation of diffuse large B-cell lymphoma. Oncol Lett 16: 882-890, 2018.

39. Gu J, Wang Y, Wang X, Zhou D, Wang X, Zhou M and He Z: Effect of the LncRNA GAS5-MiR-23a-ATG3 axis in regulating autophagy in patients with breast cancer. Cell Physiol Biochem 48: 194-207, 2018.
40. Wang X, Jiang X, Zhou L, Wang Z, Huang H and Wang M: LncRNA $\mathrm{NEF}$ is involved the regulation of gastric carcinoma cell proliferation by targeting RUNX1. Mol Med Rep 19: 2051-2056, 2019.

41. Zhang Y, Qian W, Feng F, Cao Q, Li Y, Hou Y, Zhang L and Fan J: Upregulated lncRNA CASC2 may inhibit malignant melanoma development through regulating miR-18a-5p/RUNX1. Oncol Res 27: 371-377, 2019.

42. Teng H, Wang P, Xue Y, Liu X, Ma J, Cai H, Xi Z, Li Z and Liu Y: Role of HCP5-miR-139-RUNX1 feedback loop in regulating malignant behavior of glioma cells. Mol Ther 24: 1806-1822, 2016.

43. Xu G, Wang H, Yuan D, J Yao 2 , L Meng 2 , Li K, Zhang Y, Dang $C$ and Zhu K: RUNX1-activated upregulation of lncRNA RNCR3 promotes cell proliferation, invasion, and suppresses apoptosis in colorectal cancer via miR-1301-3p/AKT1 axis in vitro and in vivo. Clin Transl Oncol 22: 1762-1777, 2020.

44. Friedman AD: Cell cycle and developmental control of hematopoiesis by Runx1. J Cell Physiol 219: 520-524, 2009.

45. Keita M, Bachvarova M, Morin C, Plante M, Gregoire J, Renaud MC, Sebastianelli A, Trinh XB and Bachvarov D: The RUNX1 transcription factor is expressed in serous epithelial ovarian carcinoma and contributes to cell proliferation, migration and invasion. Cell Cycle 12: 972-986, 2013.

46. Asciolla JJ, Renault TT and Chipuk JE: Examining BCL-2 family function with large unilamellar vesicles. J Vis Exp 68: 4291, 2012.

47. Wu D, Ozaki T, Yoshihara Y, Kubo N and Nakagawara A: Runt-related transcription factor 1 (RUNX1) stimulates tumor suppressor 533 protein in response to DNA damage through complex formation and acetylation. J Biol Chem 288: 1353-1364, 2013.

This work is licensed under a Creative Commons Attribution-NonCommercial-NoDerivatives 4.0 International (CC BY-NC-ND 4.0) License. 